\title{
Pain control, adverse effects and degree of satisfaction of postsurgical patients in the acute pain unit in a Mexican hospital using an electronic platform as a tool for assessment
}

\begin{abstract}
In Mexico, about 16 million people use health services every year. Of this group, four million are be hospitalized. $27 \%$ of hospitalized patients have an obvious surgical etiology, $60 \%$ have moderate to severe postoperative pain. ${ }^{1}$ Recovery after surgery is determined by organic dysfunction induced by stress, intestinal paralysis, cardiopulmonary complications and thromboembolism and fatigue, and patients must remain in the hospital until they reach a state of autonomous care. ${ }^{2,3}$ Advances in our understanding of pathophysiology and perioperative care suggest that several factors contribute to postoperative morbidity, length of hospital stay and convalescence, there are accelerated programs of postoperative recovery, or rapid surgery, which implies a coordinated effort to combine the preoperative education of patients, the optimization of resources, the attenuation of surgical stress, the dynamic relief of pain, mobilization and oral feeding..$^{4-7}$ Acute postoperative pain is a response to surgery and not only do patients go through unnecessary suffering, but it is also a cause of delayed discharge and a greater risk of other complications8. Risk factors for postoperative pain are usually a surgical technique, and it is likely that intraoperative nerve injury plays an important role. ${ }^{9,10}$
\end{abstract}

Volume II Issue I - 2019

\author{
Carlos Garza Garibay, Guillermo Eduardo \\ Aréchiga Ornelas, Stephanie Victoria Gómez \\ Rodríguez, Diana Romina Bucio Cedeño, Ana \\ Karen Santoyo Cueva, Ana Karen Cornejo \\ Huerta \\ Pain and paliative care unit, Hospital General de Occidente, \\ Mexico
}

Correspondence: Carlos Garza Garibay, Pain and paliative care unit, Hospital General de Occidente, Mexico, Email garza_carlosg@hotmail.com

Received: December 10, 2018 | Published: January 09, 2019

\section{Introduction}

\section{Pathophysiology}

The pathophysiology is the result of the harmful stimulation of the skin, the subcutaneous tissues, the viscera and the neuronal structures, so this is a form of exceptional acute pain. The pain occurs because inflammatory and sensitizing mediators are released that include cytokines, bradykinin and prostaglandins from injured and inflammatory cells at the site of tissue damage. Nociceptors demonstrate reversible plasticity in response to inflammatory mediators. The activation threshold of nociceptors decreases, which results in greater sensitivity to pain at the site of tissue injury. The release of ATP by the injured cells plays an important role in allodynia and the spontaneous activity by incision in the primary afferent fibers provides a maintenance of the neurons of wide dynamic range in the posterior horn in a sensitized state, as well as the repetitive stimulation of primary afferents Type $\mathrm{C}$ fibers that promote an exaggerated response to painful stimuli. ${ }^{11}$ The NMDA receptor acts through the large influx of calcium ions (Ca2p) that occurs when the channel is activated. Once inside the cell, Ca2p can activate several effectors and promote later changes. That can promote plasticity mechanisms such as long-term potentiation.

Similar plastic mechanisms are produced after acute high-intensity stimulation of C-fiber, peripheral nerve damage and inflammation, and can result in a responsiveness and high activity of the dorsal horn neurons. This phenomenon, called central sensitization ${ }^{12}$

\section{Postoperative complications}

The use of opioids and some anesthetic interventions are the best way to control or even prevent acute postoperative pain; but equally important are the adverse effects..$^{13-15}$ In anesthesiology, the different components that keep a patient hospitalized is important. Cost considerations require us to identify strategies to reduce inhospital stay. Identifying the relevant factors of the patient, anesthesia and surgery have a strong effect on the duration of the postoperative stay and is an important step to reduce the cost of care. Once these predictors are known, we can predict the duration of the postoperative stay, which also allows us to plan and provide adequate perioperative care for individual patients. However, the increase in the duration of postoperative stay associated with a given factor should be considered together with the frequency of that factor. ${ }^{16}$

The type of surgery, the choice of anesthesia, the use of opioids and interventions for the treatment of pain are risk factors for the development of adverse events, the main adverse events are sedation, nausea and vomiting, pruritus, residual blockade of the lower extremities, inactivity of the intestine or its movements and urinary retention. ${ }^{13-17}$ Post-operative nausea and vomiting (PONV) are common and distressing for patients. The overall incidence of vomiting is approximately $30 \%$, the incidence of nausea is approximately $50 \%$, and in a subset of high-risk patients, the PONV rate can be as high as $80 \% 18$. Published evidence suggests that the Universal prophylaxis of PONV is not cost-effective. Although some recommend prophylactic antiemetic therapy for high-risk patients 
and salvage antiemetic treatment for episodes of PONV, the optimal approach to managing PONV remains unclear for many physicians. ${ }^{18}$

Excessive postoperative sedation, as well as other complications, is multifactorial and is caused by the patient's physical state, the type of surgery and, in large part, by the use of opioids during the transoperative period, ${ }^{19}$ in the case of opioid sedation, the drug to reverse said effect is naloxone or naltrexone which are non-selective antagonists of the opioid receptors, but have the disadvantage of also antagonizing the analgesia mediated by them. Postoperative ileus is a concern among surgical patients. Epidural anesthesia and analgesia with local anesthetics may decrease the duration of ileus. The sparing of opioids and the use of intravenous lidocaine have been shown in some studies to improve this complication. ${ }^{20}$ Urinary retention can be a major source of anxiety and discomfort for the patient. Retention prolongs hospital stay, increases costs and can cause significant morbidity. ${ }^{21}$ In elderly patients, urinary retention can be associated with restlessness, confusion and possible delirium development. Urethral catheterization to treat postoperative retention conveys the risk of urinary tract infection, which increases each day the catheter remains in place. ${ }^{22}$

\section{Technology in medicine}

During the last two decades, the use of technology as a help tool in the different areas of health has been widely accepted, biomedical informatics is already a useful component in the different health services and biomedical research in the world, and each day is more used in hospitals and research centers for obtaining and processing data obtained from patients or projects. Although the electronic file and other data capture systems have shown to be an efficient and safe way to improve the quality of medical care, its adoption still faces resistance from the health sector. The biggest obstacle is the lack of a standardized universal system for the creation of clinical files. ${ }^{23}$

\section{Material and methods}

Transversal clinical study, prospective and observational study, 350 patients were studied who underwent surgery regardless of the type. Patients were treated by the Acute Pain Unit of our Hospital, either with IV or neuro-axial analgesia with local anesthetic and opioid. A software was made in conjunction with the Department of Pain and Palliative, Systems and Medicine of the Hospital General De Occidente, where it is possible to capture and access information in real time of post-surgical patients when they are registered on a platform, which consists of a section of the general information of the patient, the diagnosis and surgical procedure (Figure 1), type of anesthesia, choice of analgesic therapy, the most common adverse effects related to the procedure and drugs such as motor blockade, nausea, vomiting, neurological deterioration, intestinal inactivity and urinary retention (Figure 2). The analgesic efficacy with VAS scale at rest and in movement was also measured, the information of subsequent visits of each patient was captured. For intravenous analgesia, a multimodal analgesia system was used, with opioid as the basis of this and coadjuvanted with drugs that act in different pain management pathways such as: NSAID, paracetamol, lidocaine, ketamine and magnesium sulfate.

\section{Results}

In total, 350 patients were evaluated with 607 visits in total, the most common adverse effects were: motor block and intestinal inactivity in $20 \%$ and $22 \%$ (Figure 3 ).

\section{The postoperative pain}

79 of the 607 patient entries with a visual numerical scale equal to or greater than 4 at rest, which gives a result of 13\% (Figure 4). 174 of the 607 patient entries presented a visual numerical scale equal or greater than 4 in movement, which gives a result of $28 \%$ (Figure 5). Type of postoperative analgesia (Figure 6). The opioid most commonly used in intravenous analgesia was buprenorphine with $77 \%$ (270 visits) Intestinal activity according to type of postoperative analgesia (Table 1). Satisfaction was very satisfied in most patients (Figure 7).

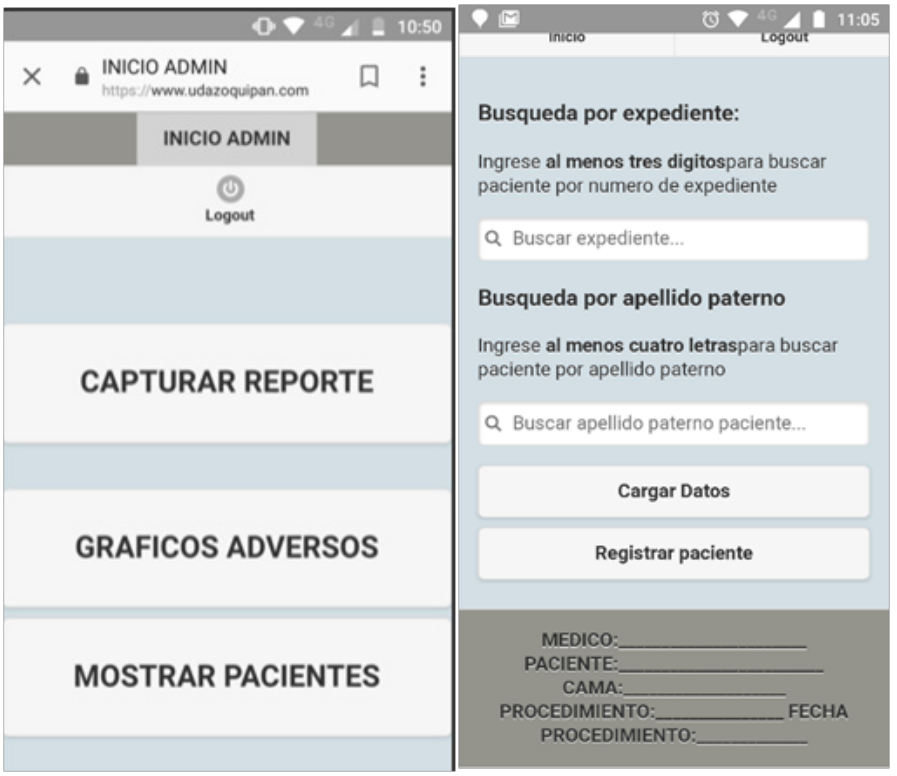

Figure I Home page for the capture of patients information in APU (Acute Pain Unit) in real time in smart device.

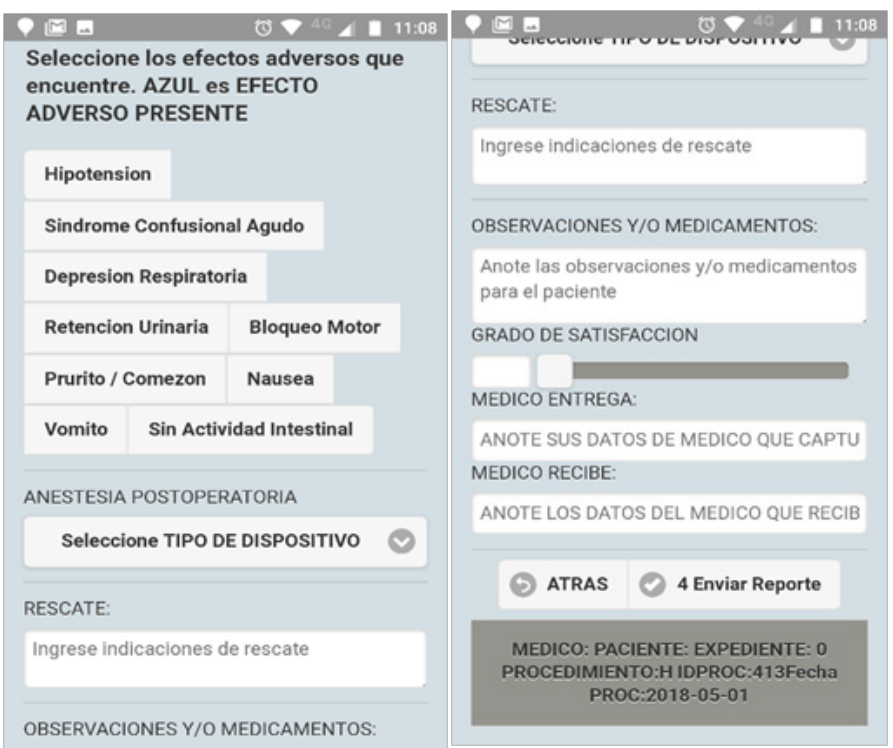

Figure 2 Selection of adverse effects and degree of satisfaction captured in real time from platform in smart device. 


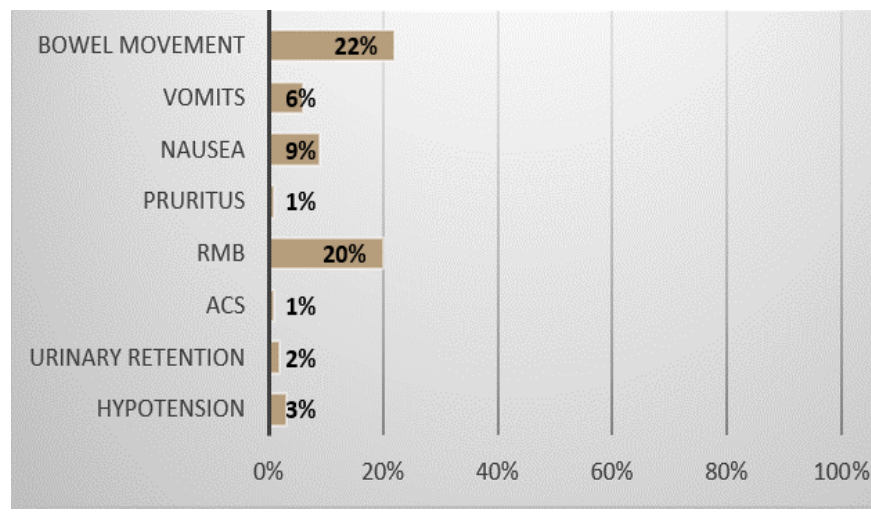

Figure 3 Frequency of adverse effects in postsurgical patients.

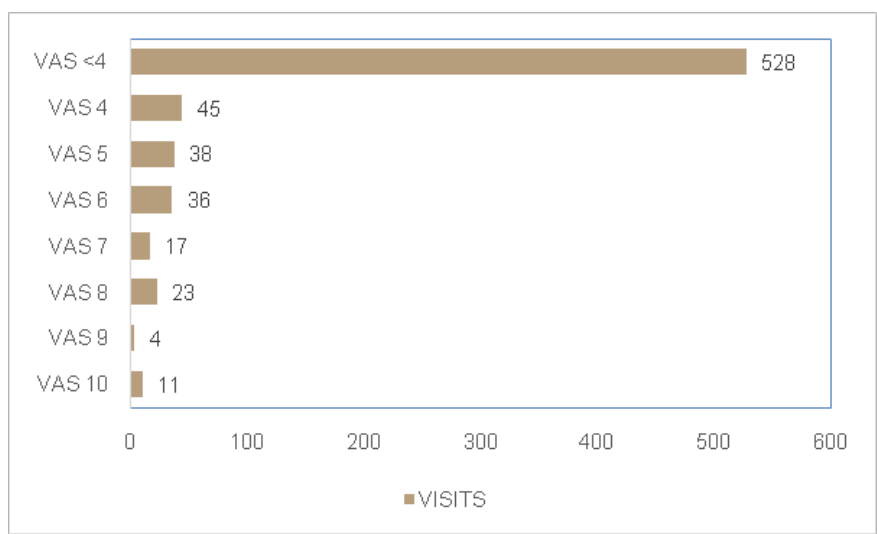

Figure 4 Pain at rest after surgery with VAS.

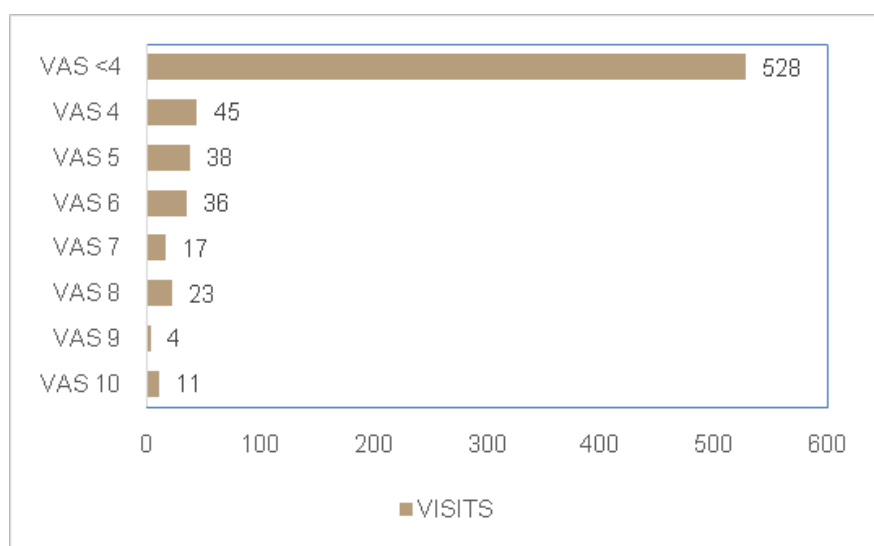

Figure 5 Pain in movement after surgery with VAS.

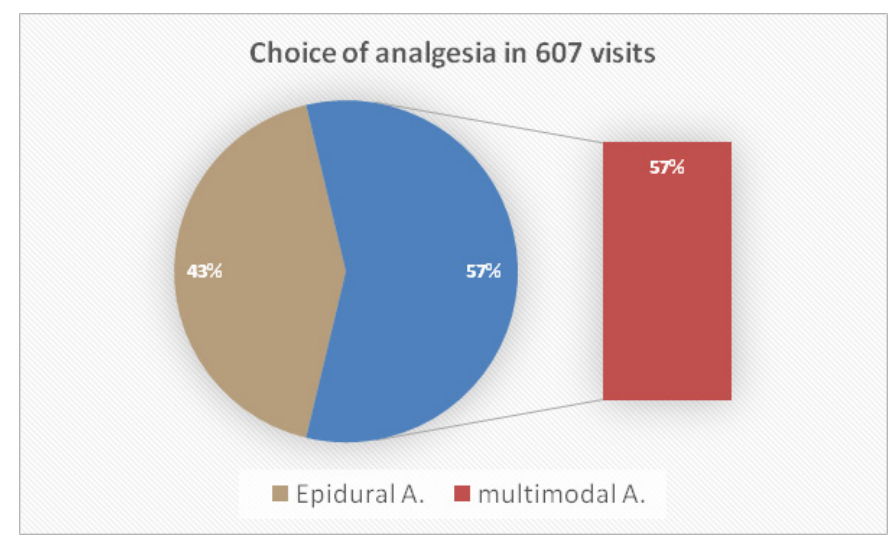

Figure 6 Choice of analgesia after surgery.
Table I Relation between choice of analgesia and bowel movement.

\begin{tabular}{|c|c|c|c|c|}
\hline & & \multicolumn{2}{|c|}{ Bowel movement } & \multirow{2}{*}{ Total } \\
\hline & & Present & Impared & \\
\hline \multirow[t]{2}{*}{ Analgesia } & Home pump & 208 & 50 & 258 \\
\hline & Opioids & 270 & 79 & 349 \\
\hline Total & & 478 & 129 & 607 \\
\hline $90 \%$ & & \multicolumn{3}{|c|}{$84 \%$} \\
\hline \multicolumn{5}{|l|}{$80 \%$} \\
\hline \multicolumn{5}{|l|}{$70 \%$} \\
\hline \multicolumn{5}{|l|}{$60 \%$} \\
\hline \multicolumn{5}{|l|}{$50 \%$} \\
\hline \multicolumn{5}{|l|}{$40 \%$} \\
\hline \multicolumn{5}{|l|}{$30 \%$} \\
\hline \multicolumn{5}{|l|}{$20 \%$} \\
\hline $10 \%$ & & $10 \%$ & & $4 \%$ \\
\hline $0 \%$ & $2 \%$ & & & $4 \%$ \\
\hline
\end{tabular}

Figure 7 Satisfaction grade of the postsurgical patients.

\section{Discussion}

The literature proposes techniques for sparing opioids and anesthetics to reduce adverse effects, $14 \%$ of the tendency is to use multimodal analgesia, which means a use of drugs and interventions that act synergistically. Synergistic interactions between different analgesics may allow lower doses of individual drugs that may provide a better safety and tolerability profile (provided there is no additivity or synergy for adverse effects). The use of 2 drugs $(A+B)$ will almost always be superior to any drug alone (ie, $\mathrm{A}+\mathrm{B}>\mathrm{A}$ and $\mathrm{A}+\mathrm{B}>\mathrm{B}$ ) and should contain agents that are effective on their own (ie, $A>$ placebo and $\mathrm{B}>$ placebo) ${ }^{24}$ Although multimodal analgesia has been described for decades, in our country they are not yet a part of clinical practice guidelines, protocols of decrease in in-hospital stay (PROSPECT or ERAS) propose pharmacological techniques and strategies that we can use, unlike the statistics of our country that we indicate that at least $60 \%$ of post-operated patients presented a pain with VAS scale greater than or equal to 4, in the acute pain unit of the Hospital General de Occidente, moderate to intense pain is significantly lower, which can explain a favorable response in the satisfaction of our patients.

According to the research cycle to have a Mexican clinical practice guide, first, several clinical studies with an acceptable sample of patients for some intervention are needed, then meta-analyzes are carried out to support this assertion and after several meta-analyzes it is possible to propose a practical guide. More studies like this are necessary to change the way anesthetists handle pain, since the commitment with the patient forces us to be updated and offer the best possible management. In our hospital, although the degree of satisfaction is high, side effects are still common and have only had a slight decrease in relation to the literature, more studies like this are needed to give more weight to patient satisfaction and empower it to demand the best possible care, the relationship between opioid use and some adverse effects was not statistically significant, several reasons may be responsible, in our unit we use low doses of opioids when adding adjuvants such as Lidocaine, Ketamine, Magnesium Sulfate, NSAID and Paracetamol among others, also perhaps biased by anesthetic and surgical techniques. Progress speaks of a state of improvement of the human condition and technology not only improves the daily life of people, it is imperative to advance in medicine to more practical and efficient systems, although some 
doctors and health systems refuse to evolve to electronic platforms, the efficiency of these is not in doubt and sooner or later we will migrate to these systems. The platform developed for the management of postoperated patients is useful thanks to its practicality and reliability in the safeguarding of information using smart devices in real time.

\section{Acknowledgments}

None.

\section{Conflicts of interest}

Author declares that there is no conflicto of interest.

\section{References}

1. Covarrubias gómez A. El manejo del dolor agudo postoperatorio: una década de experiencias. 2013;36:179-182.

2. Basse L, Jakobsen DH, Billesbølle P, et al. A Clinical Pathway to Accelerate Recovery After Colonic Resection. 2000;232(1):51-57.

3. Bardram L, Funch Jensen P, Jensen P, et al. Recovery after laparoscopic colonic surgery with epidural analgesia and early. Lancet. 1995:345(8952):763-764.

4. Kehlet H, Dahl JB. Anaesthesia, surgery and challenges in postoperative recovery. Lancet. 2003;362(9399):1921-1928.

5. Kehlet H, Wilmore DW, DM. Multimodal strategies to improve surgical outcome. Am J Surg. 2002;183(6):630-641.

6. Miller KR, Wischmeyer PE, Taylor B, et al. An evidence-based approach to perioperative nutrition support in the elective surgery patient. JPEN J Parenter Enteral Nutr. 2013;37(5 Suppl):39S-50S.

7. Ljungqvist O. Jonathan E. Rhoads lecture 2011: Insulin resistance and enhanced recovery after surgery. JPEN $J$ Parenter Enteral Nutr 2012;36(4):389-398.

8. Lovich-sapola J, Smith CE, Brandt CP. Postoperative pain control. Surg Clin North Am. 2015;95(2):301-318.

9. Reddi D, Curran N. Chronic pain after surgery : pathophysiology, risk factors and prevention. Postgrad Med J. 2014;90(1062):222-227.

10. Maguire MF, Ravenscroft A, Beggs D, et al. A questionnaire study investigating the prevalence of the neuropathic component of chronic pain after thoracic surgery. Eur J Cardiothorac Surg. 2006;29(5):800-805.
11. Mello RD, Dickenson AH. Spinal cord mechanisms of pain. $B r J$ Anaesth. 2008;101(1):8-16.

12. Woolf CJ, Ma Q. Review Nociceptors-Noxious Stimulus Detectors Neuron. 2007;55(3):353-364.

13. Ed M, Mc F, Hudcova J. Patient controlled opioid analgesia versus nonpatient controlled opioid analgesia for postoperative pain. Cochrane Database Syst Rev. 2015;(6):CD003348.

14. Kehlet H, Wilkinson RC, Fischer HB, et al. PROSPECT: evidencebased, procedure-specific postoperative pain management. Best Pract Res Clin Anaesthesiol. 2007;21(1):149-159.

15. Chou R, Fanciullo GJ, Fine PG, et al. Clinical Guidelines for the Use of Chronic Opioid Therapy in Chronic Noncancer Pain. J Pain. 2009;10(2):113-130

16. Chung F, Mezei G. Factors Contributing to a Prolonged Stay After Ambulatory Surgery. Anesth Analg. 1999;89(6):1352-1359.

17. Zhang Z, Mostofian F, Ivanovic J, et al. All grades of severity of postoperative adverse events are associated with prolonged length of stay after lung cancer resection. $J$ Thorac Cardiovasc Surg. 2018;155(2):798-807.

18. Gan TJ, Diemunsch P, Habib AS, et al. Consensus Guidelines for the Management of Postoperative Nausea and Vomiting. 2014;118(1):85-113.

19. Memis D, Turan M, Kavalci G, et al. Intravenous paracetamol reduced the use of opioids, extubation time, and opioid-related adverse effects after major surgery in intensive care unit. J Crit Care. 2010;25(3):458-462.

20. Groudine SB, Fisher HAG, Kaufman RI, et al. Intravenous Lidocaine Speeds the Return of Bowel Function, Decreases Postoperative Pain, and Shortens Hospital Stay in Patients Undergoing Radical Retropubic Prostatectomy. Anesth Analg. 1998;86(2):235-239.

21. Tammela T, Kontturi M, Lukkarinen O. Postoperative Urinary Retention : I . Incidence and Predisposing Factors. Scand J Urol Nephrol. 1986;20(3):197-201.

22. Darrah DM, Griebling TL, Silverstein JH. Postop erative Urinar y Retention. Anesthesiol Clin. 2009;27(3):465-484.

23. Luis J, Vázquez T. El expediente clínico electrónico. Revista odontológica mexicana. 2010;14(2):76-77.

24. Eisenberg E, Suzan E. Drug Combinations in the Treatment of Neuropathic Pain. Curr Pain Headache Rep. 2014;18(12):463. 